\title{
Jährliches Mammografiescreening ist nicht nötig
}

\begin{abstract}
$A b$ welchem Alter und in welchen Zeitabständen sollten Frauen zur Brustkrebsfrüherkennung mammografiert werden? In Deutschland gelten seit Januar 2004 die EU-Richtlinien. Demnach haben Frauen zwischen 50 und 69 Jahren Anspruch auf eine Mammografieuntersuchung, die alle zwei Jahre wiederholt wird. Nach einer neuen US-Studie scheint dies derzeit die optimale Strategie zu sein.
\end{abstract}

— In Teilen der USA wird die Mammografie Frauen ab 40 im jährlichen Abstand empfohlen. Eine Forschungsgruppe hat jetzt sechs verschiedene Screeningstrategien analysiert. Varianten waren Alter der Teilnehmerinnen und die Zeitabstände zwischen den Screeninguntersuchungen. Messdaten waren die Zahl der Mammogramme, die Reduktion der Brustkarzinommortalität durch das Screening, falsch po- sitive Resultate, unnötige Biopsien und Überdiagnose.

Die sechs Modelle produzierten ein konstantes Ranking: Die Mammografie im Zweijahresabstand erreichte 81\% (67\%-99\%) der Trefferquote der jährlichen Untersuchung, aber mit nur halb so viel falsch positiven Befunden. Auch der Screeningbeginn ab 50 Jahren gegenüber 40 Jahren bewährte sich. Das Screening der Frauen von 5069 Jahren reduzierte die Mortalität um $16,5 \%(15 \%-23 \%)$ gegenüber einem Nichtscreening. Die zweijährige Mammografie ab 40 (versus 50 ) verminderte die Mortalität um weitere 3\% (1\%$6 \%)$. Dem stehen mehr falsch positive Befunde gegenüber, die mit überflüssigen Biopsien und unnötiger psychischer Belastung einhergingen. Das zweijährige Screening über das 69. Lebensjahr hinaus reduziert die Mortalität weiter, aber parallel nimmt die Überdiagnostik erheblich zu.
KOMMENTAR: Frauen zwischen 50 und 69 Jahren profitierten am meisten von einer Mammografie im Zweijahresabstand. Denn besser als beim einjährigen Screening hielten sich hier die Chance, ein Brustkarzinom frühzeitig zu entdecken, oder das Risiko von falsch positiven Befunden die Waage. Eine Mammografie alle zwei Jahre entspricht auch der Kinetik der Entwicklung des Brustkarzinoms. Meistens entwickelt es sich so langsam, dass der Unterschied zwischen dem einund zweijährigen Mammografiescreening das Überleben der Patienten nicht beeinflusst. Für Frauen mit einem aggressiven, schnell wachsenden Brustkarzinom verbessert das einjährige Screening die Überlebenschancen kaum.

K. MALBERG

\section{- J. S. Mandelblatt et al.}

Effects of mammography screening under different screening schedules: model estimates of potential benefits and harms. Annals Int. Med. 151 (2009) 738-747

\section{Schwarze Nägel}

— Eine 80-jährige Frau störte sich an der streifenförmigen Pigmentierung beider Daumennägel (Pfeile), die sie seit mehr als einem Jahr nach Beginn einer Behandlung mit Hydroxyurea wegen einer essenziellen Thrombozytämie bemerkt hatte. Man stellte die Diagnose einer longitudinalen Melanonychie. Obwohl grundsätzlich alle Nägel betroffen sein können, waren bei dieser Frau nur die beiden Daumennägel verfärbt.

Zum Zeitpunkt der Vorstellung nahm die Patientin keine weiteren Medikamente ein, die zu Nagelverfärbungen führen können, auch bestanden keine Symptome der Grund-

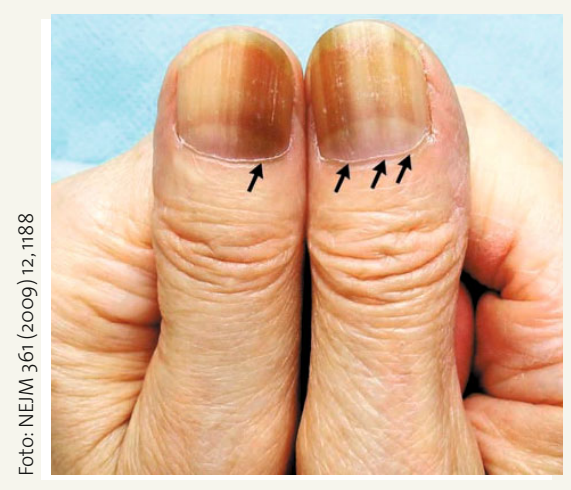

erkrankung. Die Plättchenzahl betrug 420000 pro Mikroliter. Die Melanonychie ist eine seltene Nebenwirkung einer Hydroxyureatherapie. Die Differenzialdiagnosen von
Nagelverfärbungen beinhalten ein subunguales Melanom, ein pigmentiertes Plattenepithelkarzinom, ein subunguales Hämatom, ein Nävus oder eine Pigmentierung durch andere Medikamente, z.B. Cyclophosphamid, Doxorubicin, Minocyclin oder Zidovudin. Auf welche Weise Hydroxyurea diese Verfärbungen herbeiführt, ist unbekannt. H. S. FÜEßL =

- D. Ranta, C. Bonmati (Centre Hospitalier Universitaire NancyBrabois, Vandoeuvre-les-Nancy, France). Acquired melanonychia. New Engl. J. Med. 361 (2009) 12, 1188 